\title{
TAB3 0-GIcNAcylation promotes metastasis of triple negative breast cancer
}

\author{
Tao Tao ${ }^{1,2}$, Zhixian $\mathrm{He}^{3}$, Zhiming Shao ${ }^{1}$, Haojie Lu' ${ }^{1,2,4}$ \\ ${ }^{1}$ Shanghai Cancer Center and Institutes of Biomedical Sciences, Fudan University, Shanghai 200032, P.R. China \\ ${ }^{2}$ Department of Chemistry, Fudan University, Shanghai 200433, P.R. China \\ ${ }^{3}$ Department of General Surgery, Affiliated Hospital of Nantong University, Nantong 226001, P.R. China \\ ${ }^{4}$ Key Laboratory of Glycoconjugates Research Ministry of Public Health, Fudan University, Shanghai 200032, P.R. China
}

Correspondence to: Haojie Lu, e-mail: luhaojie@fudan.edu.cn

Keywords: triple negative breast cancer, metastasis, TAB3, O-glcNAcylation

Received: October 08, 2015

Accepted: January 29, 2016

Published: March 18, 2016

\section{ABSTRACT}

O-GIcNAcylation is a post-translational modification that regulates a broad range of nuclear and cytoplasmic proteins and is emerging as a key regulator of various biological processes. Although previous studies have shown that increased levels of global O-GIcNAcylation and O-GIcNActransferase are linked to the incidence of metastasis in triple negative breast cancer (TNBC) patients, the molecular basis behind this is not fully understood. In this study, we have determined that the TAK1 binding protein 3 (TAB3) was O-GIcNAcylated at Ser408 by OGT in the TNBC, which was required for its Thr404 phosphorylation, TAK1 activation and downstream nuclear factor kappa B (NF-kB) activation in TNBC. O-GIcNAcylation of TAB3 was induced by p38 MAPK and it in turn enhances the TAK1 mediated p38MAPK activation, which forms the positive feedback loop in TAB3mediated NF-KB activation. In TNBC, TAB30GIcNAcylationmediated cell migration and invasion by activating its downstream NF-KB. The expression of TAB3 O-GICNAcylation increased in TNBC patients, and it was significantly correlated with poor prognoses of the patients. Our study provides insights into the mechanism of TAB3 regulating activity and suggests its important implications in TNBC metastasis.

\section{INTRODUCTION}

Breast cancer is becoming the main causes of cancer death among women. Breast cancer is classified into 4 subtypes, including Lumina A, Lumina B, HER-2+ and Basel-like (also termed triple negative breast cancer, TNBC), based on the molecular characteristics of the cancer genetics. TNBC is the most prevalent one which remains incurable despite of recent therapeutic advances [1]. It was known that chronic inflammation play an essential role in cancer development and metastasis. The major link between chronic inflammation and breast cancer metastasis is mediated by activation of nuclear factor- $\kappa \mathrm{B}(\mathrm{NF}-\kappa \mathrm{B})[2]$. For example, pro-inflammatory cytokine interleukin-1 $\beta$ (IL-1 $\beta$ ), which is secreted by adipose tissue or macrophages, promotes breast cancer metastasis by constitutive and deregulated activation of NF- $\kappa \mathrm{B}$ signaling pathway [3].
During the cytokine induced activation of $\mathrm{NF}-\kappa \mathrm{B}$, inhibitory subunit $\mathrm{IkB} \alpha$ undergoes phosphorylation, ubiquitylation and degradation, leading to nuclear translocation of the RelA (p65)-RelB (p50) complex, where it activates transcription of target genes [4]. Considerable progress has been made in the past couple of decades in identifying molecular compounds involved in cytokine triggered NF- $\kappa \mathrm{B}$ activation pathway. TAK1 binding proteins (TABs) is the major adaptor protein family in the $\mathrm{NF}-\kappa \mathrm{B}$ activation, in which linked the TRAFs molecular via ubiquitin chain to activate Transforming growth factor $\beta$ activated kinase 1 (TAK1) [5]. TABs family contains three members, including TAB1, TAB2 and TAB3. Unlike the TAB1, TAB2 and TAB3 bind to the TRAFs protein via their C-terminal nuclear zinc finger (NZF) motif, which leads to the autophosporylation and activation of TAK1 [6]. Previous study has demonstrated that TAB1 is not expressed in breast 
cancer cells. And TAK1-TAB2 signal axis is important for cytokine mediated metastasis of breast cancer [7]. TAB2 and TAB3 are homologs which have redundant functions in mediating IL- $1 \beta$ or TNF- $\alpha$ signaling [8]. However, whether TAB3 is involved in breast cancer metastasis is enigmatic.

Protein O-GlcNAcylation is an abundant posttranslational modification of serines/threonines occurring on nuclear and cytoplasmic proteins. O-GlcNAcylation is implicated in various cellular processes, including protein turnover, gene transcription, cellular responses to insulin, cell-cycle control, stress protection and calcium cycling [9]. The enzymes responsible for the attachment (O-GlcNActransferase, OGT) and removal(OGlcNAcase, OGA) of this sugar moiety have been found in the nucleus and the cytoplasm of cells [10]. Similar to phosphorylation, modification by O-GlcNAc is dynamic, giving rise to functionally distinct protein species. There is also evidence suggested that O-GlcNAc may interplay with protein phosphorylation [11]. Dysfunctional protein O-GlcNAcylation/phosphorylation appears to have a role in the pathology of type II diabetes, Alzheimer's disease, inflammation, and cancer development. It has been reported recently that increased levels of global GlcNAcylation and OGT are closely linked to the metastasis of breast cancer [12-14]. In the present study, we explored the role of TABs O-GlcNAcylation in breast cancer metastasis. Previous study has reported O-GlcNAcylation of TAB1 modulated TAK1-mediated cytokine release [7]. Since TAB1 is not expressed and TAB2 in not O-GlcNAcylated in breast cancer by our previous results, the present study focus on whether TAB3 O-GlcNAcylationis participating in the metastasis of breast cancer.

The present study demonstrates that O-GlcNAcylation of a single residue (Ser408) on TAB3 modulates TAK1 activation in response to IL-1 $\beta$ stimulation. TAB3 O-GlcNAcylation promotes breast cancer metastasis by activating NF- $\kappa$ B signal transduction. Our findings provide insight into the regulatory mechanism of TAB3 function and reveal the implication of aberrant TAB3 O-GlcNAcylation in cancer metastasis.

\section{RESULTS}

\section{OGT promoted TNBC cell migration and invasion in a TAB3 dependent manner}

To examine the expression profiles of TABs in breast cancer, the expression of TAB1, TAB2 and TAB3 in 5 breast cancer cell lines, including MCF-7, T47D, SK-BR-3, MDAMB-231, MDA-MB-468 were detected by Western Blot. TAB1 was undetected in all cells, but TAB2were expressed in all five cell lines. Among these cell lines, TAB3 were expressed in 2 TNBC cell lines including MDA-MB-231 and MDA-MB-468 cells (Figure S1A). MDA-MB-231 cells show higher intracellular TAB3 expression then MDA-MB-468 cells, whereas MCF-7, T47D and SK-BR-3 had rarely detectable TAB3 (Figure S1A). To investigate whether the expression of TAB3 was associated with breast cancer cell migration and invasion, stable MDA-MB-231 or MDA-MB-468 cells transfected with TAB3 expression or knockdown plasmid were established (Figure 1A). MTT assay showed neither knockdown nor overexpression ofTAB3 impacted cell viabilities (Figure S1B), essentially ruling out a possible role for TAB3 in regulating cell survival. On the other hand, wound healing assay and transwell assay indicated that the migratory and invasion capacity of MDA-MB-231 transfected with siRNA targeting TAB3 was significantly decreased compared to cells transfected with non-specific siRNA, whereas it was increased inMDA-MB-468 cells ectopic over-expressing TAB3 (Figure 1B-1C, Figure S2). To test whether the association between OGT expression and breast cancer cell migration and invasion were mediated by TAB3, the breast cancer cellsMDA-MB-231were transfected with ectopic human OGT and TAB3siRNA. Silencing of TAB3 in OGToverexpressing cells almost entirely reversed OGT-enhanced cell migration and invasion (Figure 1D, Figure S3). These results suggested that OGT promoted TNBC cell mobility and may involve in modulation of TAB3 O-GlcNAcylation.

\section{TAB3was O-GIcNAcylated at Ser408 in TNBC cells}

To identify whether TAB3 was O-GlcNAcylated in breast cancer cells, we used several approaches to detect its O-GlcNAc modification. First, prokaryotic expressed TAB3 was O-GlcNAcylated in vitro. The TAB3 O-GlcNAcylation was examined by the anti-O-GlcNAc antibody CTD110.6 (Figure 2A). This was further confirmed by the chemoenzymatic labeling of the O-GlcNAc residue in which the O-GlcNAc moiety on the protein is labeled with UDP-GalNAz using a mutant galactosyltransferase GalT1 Y289L (mGalT1) with an azidederivative of UDP-GalNAc (UDP-GalNAz) as donor substrate, followed by labeling with biotin alkyne [15]. After in vitro O-GlcNAcylation, TAB3 was subjected to $\mathrm{mGalT} 1$ labeling and then detected by probing with streptavidin-conjugated HRP (Figure 2B). To confirm TAB3 as a bona fide OGT substrate with dynamic O-GlcNAc modification in vivo, we also detect GlcNAcylation of Flag-TAB3 using the O-GlcNAc-specific antibody CTD110.6 in MDA-MB-231 cells. As a specificity control, we performed a reductive $\beta$-elimination reaction under mild alkaline conditions to remove O-GlcNAc. As expected, $\beta$-elimination treatment completely abolished the O-GlcNAc signal on TAB3 (Figure 2C). To further confirm the O-GlcNAc signal on TAB1, TAB1 immunoprecipitated from MDA-MB-231 cells was treated with GlcNAcstain [16], a inhibitor of OGA, which increased O-GlcNAcylation of TAB3 (Figure 2D). O-GlcNAcylation is known to occur at Thr and Ser residues, and TAB3 consists of $13 \mathrm{Ser} / \mathrm{Thr}$ residues. To identify O-GlcNAcylated sites on TAB3, 
immunoprecipitated and subjected to electron transfer dissociation (ETD)-MS analysis were used. We found that the Ser 408 is the signal O-GlcNAc site on TAB3 (Figure 2E). Furthermore, OGT was cotransfected with wildtype or S408A mutant TAB3. The impact of OGT over expressionon the O-GlcNAcylation of both constructs was assessed. Overexpression of OGT apparently increased O-GlcNAcylation in wild-type TAB3, which was largely blocked by OGT siRNA, but barely affected the S408A mutant. These results suggested that Ser-408 is the major O-GlcNAcylation site on TAB3 (Figure 2F).

\section{O-GIcNAc modification of TAB3 modulated TAK1 activation in TNBC cells}

Previous study show that TAB3 is essential in IL-1 $\beta$ mediated signal transduction cascades, which trigger TAK1 activity and subsequent activation of the transcription factor NF- $\kappa \mathrm{B}$ [17]. To investigate the effects of O-GlcNAcylation of TAB3 on activation of the TAK1 kinase and downstream signaling, we examined the O-GlcNAcylation of TAB3 in MDA-MB-231 cells. The site specific O-GlcNAc antibody, which recognized the S408 O-GlcNAcylation,
A

MDA-MB-468

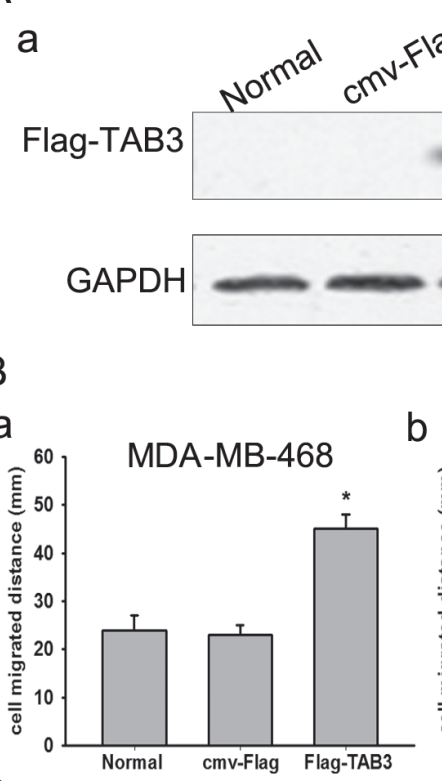

D

a

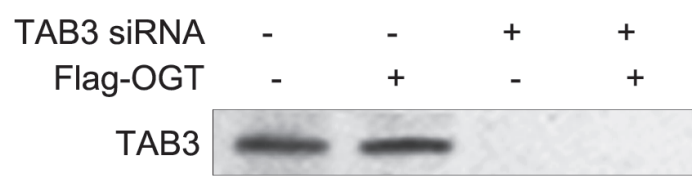

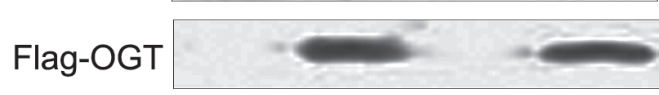

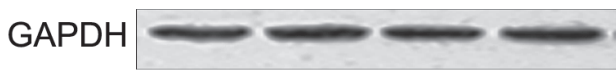

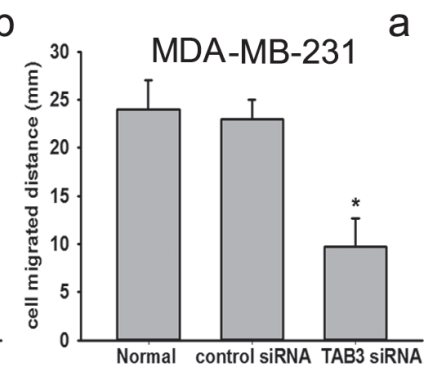

C

a
MDA-MB-231
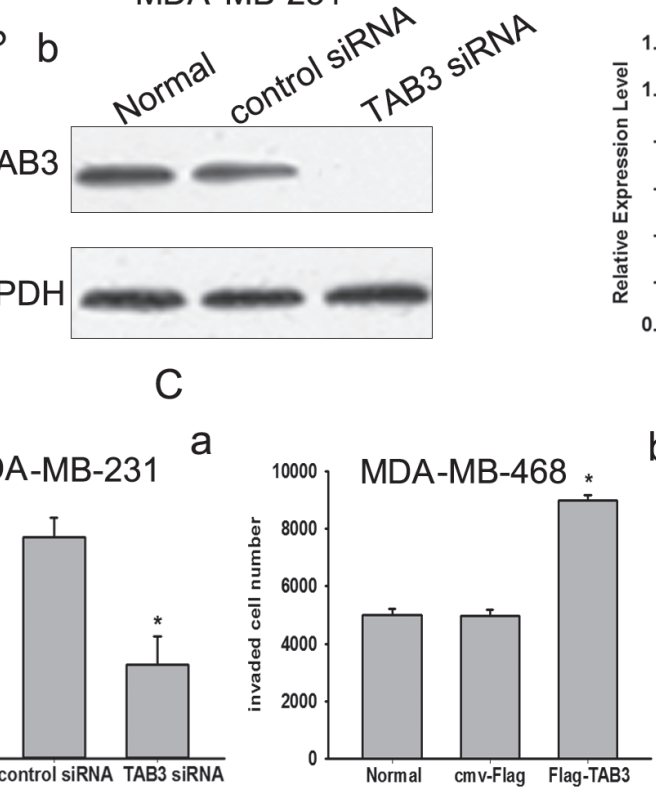

b
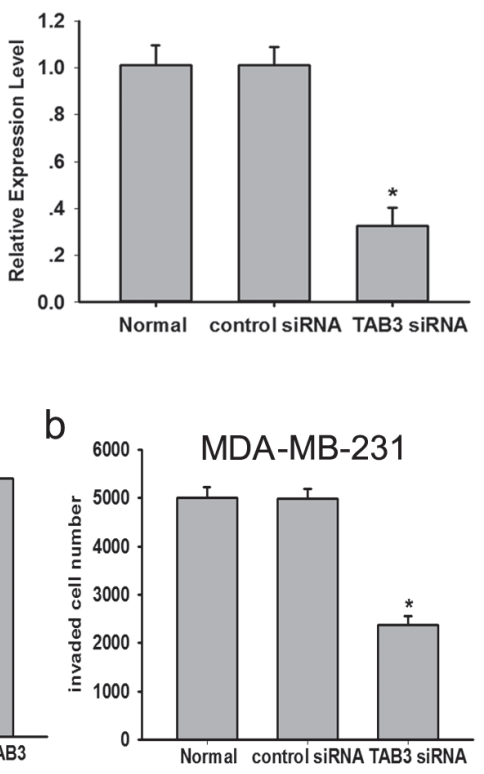

Normal control siRNA TAB3 siRNA b

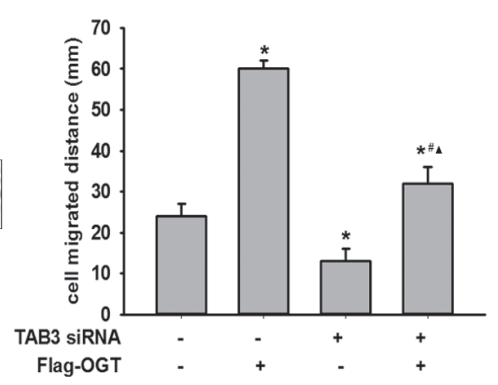

C

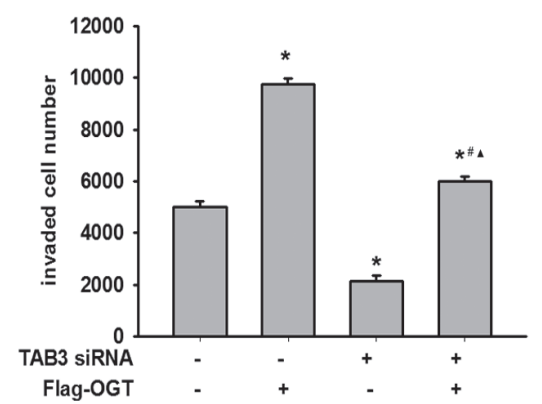

Figure 1: TAB3 involved in OGT promoted TNBC cells migration and invasion. (A) Protein expression of TAB3 analyzed by Western Blot. MDA-MB-468 cells were transfected with the cmv-Flag and Flag-TAB3 expression vector as indicated (a). MDA-MB-231 cells were transfected with the control siRNA and TAB3 siRNA vector as indicated (b). The data were means \pm SEM. $* P<0.05$, compared with the untransfected normal group. (B) Equal numbers of indicated stably transfected MDA-MB-468 cells (a) and MDA-MB-231 cells (b) seeded into six-well tissue culture plates. Wound healing assay was performed and analyzed as described under Methods. The data were means \pm SEM. ${ }^{*} P<0.05$, statistically significant compared with the normal. (C) $10^{6}$ stably transfected MDA-MB-468 cells (a) and MDA-MB-231 cells (b) cultured in matrigel chambers. Transwell assay was performed and analyzed as described under Methods. The data were means \pm SEM. ${ }^{*} P<0.05$, compared with the normal group. (D) MDA-MB-231 cells were transfected with Flag-OGT and/or TAB3 siRNA vector. After $48 \mathrm{~h}$, protein expressions of TAB3 and Flag-OGT were analyzed by western blot (a). Cell migration (b) and invasion (c) were assessed as described. The data were means \pm SEM. $* P<0.05$, statistically significant compared with the untransfected control group; ${ }^{\#} P<0.05$, statistically significant compared with OGT over-expression group; ${ }^{\mathbf{\Delta}} P<0.05$, statistically significant compared with the TAB3 siRNA transfected group. 
was generated using the classical approach (Figure S4A). The O-GlcNAc modification of TAB3 and the activation of TAK1 increased during IL-1 $\beta$ stimulation (Figure S4B-S4C). In addition, we introduced WT TAB3 and the O-GlcNAc-deficient S408A mutant into MDA-MB-231 cells. When transfected with WT TAB1, IL-1 $\beta$ induced TAK1 activation and its phosphorylation increased (Figure 3A and 3B).In contrast, IL-1 $\beta$ induced TAK1 kinase activity and its phosphorylation reduced in the O-GlcNAcdeficient TAB3 S408A mutant (Figure 3A and 3B). To investigate the effect of TAB3 O-GlcNAcylation on the TAK1 downstream signaling, NF- $\mathrm{B}$ activation andp38 MAPK signaling pathway activation were examined. In cells transfected with O-GlcNAc-deficient

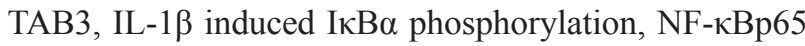
phosphorylation, and $\mathrm{NF}-\kappa \mathrm{B}$ luciferase activity were decreased compared to cells transfected with WT TAB3 (Figure 3C and 3D). The phosphorylation of p38 in S408A mutant cells decreased compared to cells transfected with the WT TAB3, (Figure 3E).

\section{O-GIcNAcylation of TAB3 at Ser408 was dependent for its phosphorylation at Thr404}

Protein O-GlcNAcylation interplays with protein phosphorylation. TAB3 contains three characterized phosphorylation sites that are modified byIL-1 $\beta$ stimulation and are involved in TAK1 activation [18]. To investigate the possible effect of TAB3 O-GlcNAcylation on its phosphorylation, the WT and S408A mutant TAB3 were transfected into MDA-MB-231 cells. When stimulated by IL-1 $\beta$, phosphorylated T404 TAB3 increased in WT transfected cells, but not in S408 mutant transfected cells.(Figure 4A). Previous studies have reported that $\mathrm{TAB} 3$ promotes the signal transduction by binding to the ubiquitin chain of TRAF6 [19]. In WT TAB1 transfected cells, the binding of TAB3 with K63 linked polyubiquitin chain of TRAF6 and the binding of TAB3 with TAK1increased withIL-1 $\beta$ stimulation, however the binding was no affected in cells transfected with the S408A mutant TAB3 (Figure 4B). To further confirmed the interaction between O-GlcNAcylation and phosphorylation in TAB3, the WT and T404A mutant TAB3 was constructed and transfected into cells. The T404A mutant TAB3 has no effect on TAB3 O-GlcNAcylation in both IL-1 $\beta$ treated or untreated cells compared with WT TAB3 (Figure 4C). TAB3 showed increased phosphorylation of T404 in WT compared with the S408A mutant, while there was no change on S60 or S506 phosphorylation. TAB3 T404 is phosphorylated by p38 pathway. To investigate the role of p38 pathway in TAB3 phosphorylation and O-GlcNAcylation, cells were

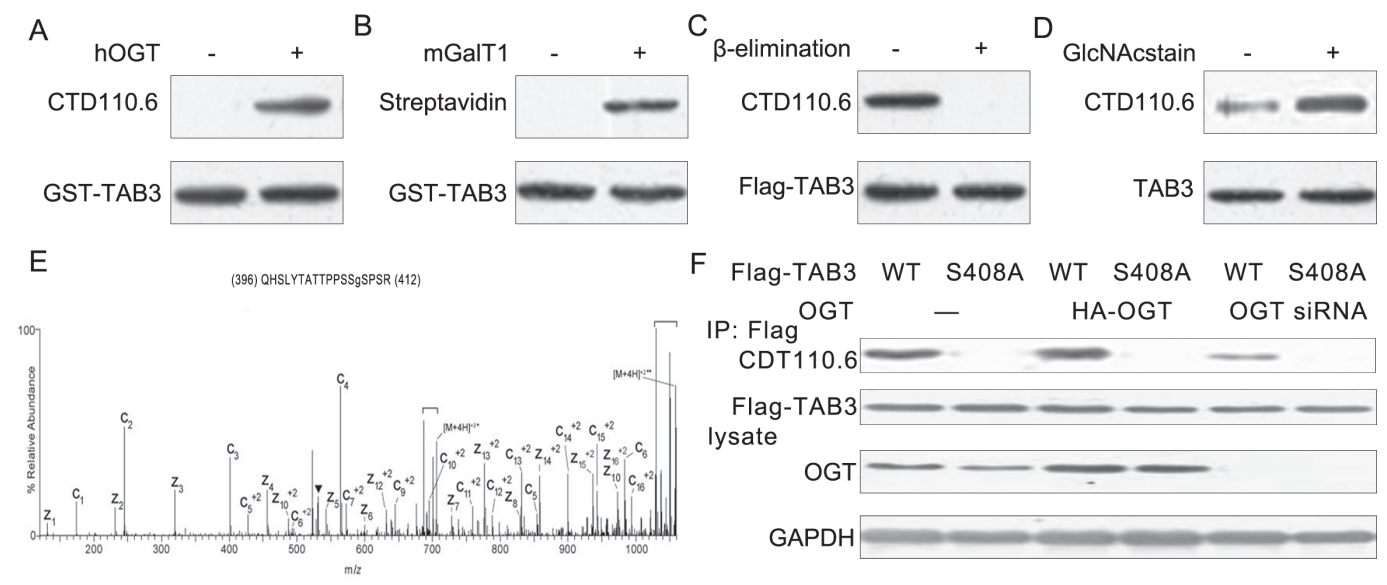

Figure 2: TAB3 is O-GIcNAcylated on Ser408 in TNBC cells. (A) Prokaryotic expressed TAB3 was incubated with recombinant hOGT. The samples were subjected to SDS-PAGE and immunoblotted with a generic O-GlcNAc antibody CTD110.6. Total protein was detected with the Flag antibody as a loading control. (B) Chemoenzymatic labeling of the O-GlcNAc residue In vitro O-GlcNAcylated TAB1 was subjected to enzymatic labeling using galactosyltransferase (mGalT1) labeling and UDP-GalNAz, before reacting with biotin alkyne for detection with HRP. The samples were subjected to SDS-PAGE and probed with horseradish peroxidase conjugated streptavidin (Extravidin-HRP). (C) Lysates from HEK293 cells transfected with cmv-Flag or Flag-TAB3 were immunoprecipitated for Flag antibody and treated with $\beta$-elimination. The samples were subjected to SDS-PAGE and probed with CTD110.6 antibody. (D) In vivo O-GlcNAcylation of TAB3 was detected by immunoprecipitating the endogenous TAB3 from MDA-MB-231 cells (treated with or without $1 \mathrm{mM}$ GlcNAcstatin) using an antibody directed against TAB3. Immunoprecipitates were subjected to SDS-PAGE and immunoblotted with CTD110.6 and then with the TAB3 antibody for loading controls. (E) An ETD MS/MS spectrum recorded on $[\mathrm{M}+4 \mathrm{H}]^{+4}$ ions of GlcNAcylated peptide QHSLYTATTPPSSSPSR. An ETD-enabled LTQ mass spectrometer was operated followed by four data-dependent scans after every MS1 scan to generate the ETD spectrum, which is presented as a subtracted spectrum. Predicted product c'- and z'- ions are listed above and below the peptide sequence, respectively. Single- and double-charged ions are listed as monoisotopic and average masses, respectively. Observed product ions are underlined and are sufficient to define the O-GlcNAc residue at Ser-408. (F) MDA-MB-231 cells were cotransfected with FLAG-tagged wild-type or S408A mutant TAB3 along with OGT siRNA vector for $48 \mathrm{~h}$. TAB3 was enriched and the O-GlcNAcylation of TAB3 was detected using antibody CTD110.6. 
pre-treated with the p38 inhibitor SB203580. Inhibition of p38 activation diminished TAB3 O-GlcNAcylation and phosphorylation in both IL-1 $\beta$ treated or untreated cells (Figure 4D). These results suggest that O-GlcNAc at Ser408 may regulate TAB3 phosphorylation at T404.

\section{TAB3 O-GIcNAcylation promoted TNBC cell migration and invasion in vitro and in vivo}

Previous studies have demonstrated TAB3 promote breast cancer metastasis [7]. To examine whether the promotion of cell migration and invasion induced by TAB3 was mediated by TAB3 O-GlcNAcylation, the WT and S408A mutant TAB3 expression vector were stably transfected into MDA-MB-231 cells. S408A mutant TAB3 over-expression reversed the WT TAB3 over-expression induced cell migration and invasion both in cells treated or untreated with IL-1 $\beta$ (Figure 5A-5B, Figure S5). To further investigate the role of TAB3 O-GlcNAcylation in breast cancer migration and invasion in vivo, the in situ breast cancer model was used. Consistent with the findings in vitro, injection of TAB3 over-expression MDA-MB-231
A

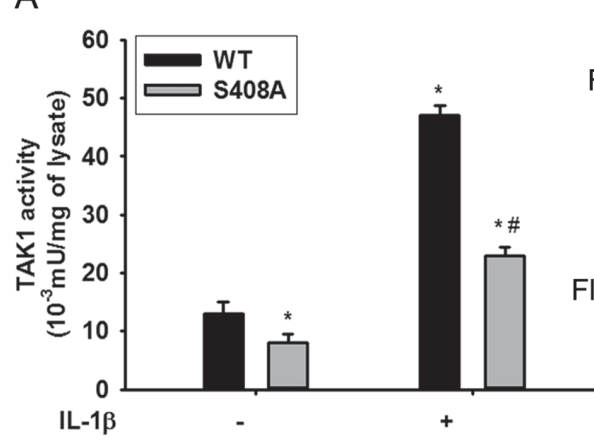

C

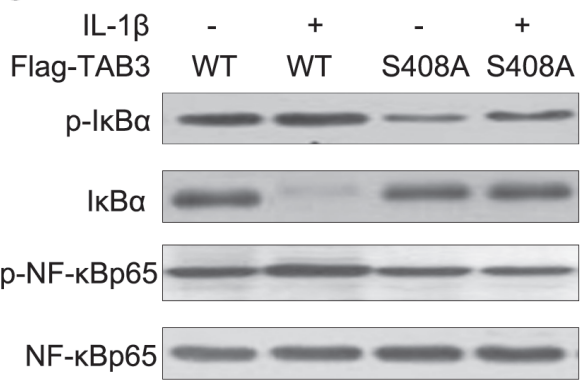

D

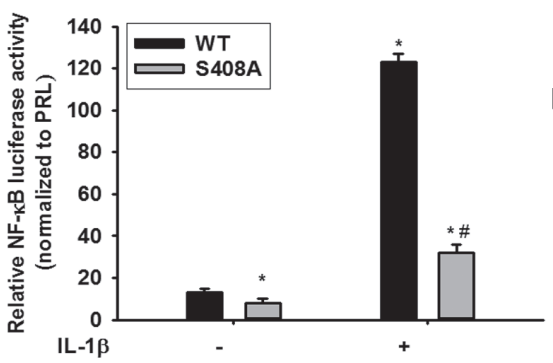

$\mathrm{B}_{\mathrm{IL}-1 \mathrm{~B}-+}-\quad+$

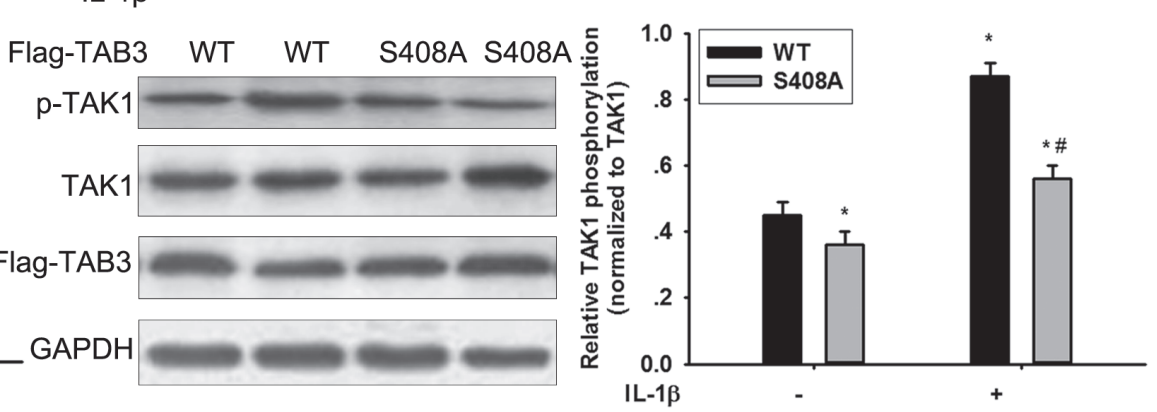

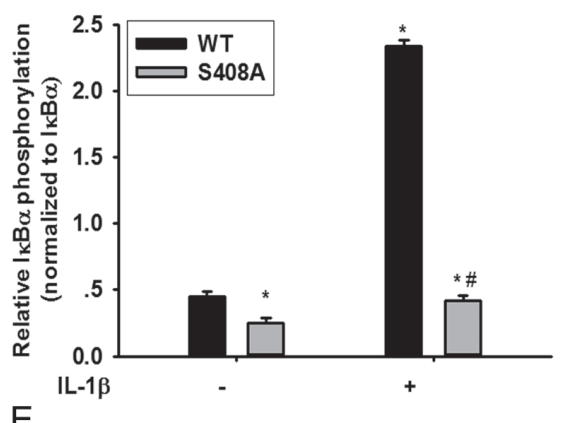

E

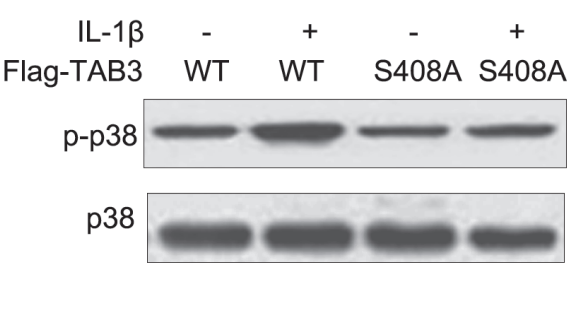

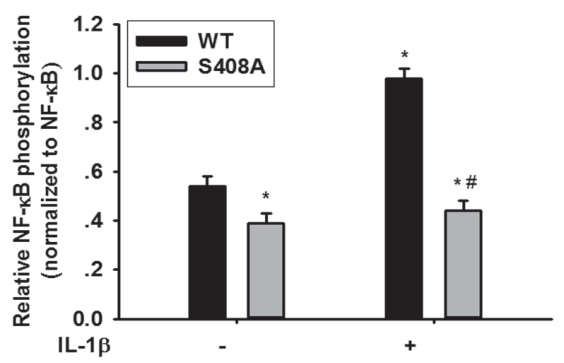

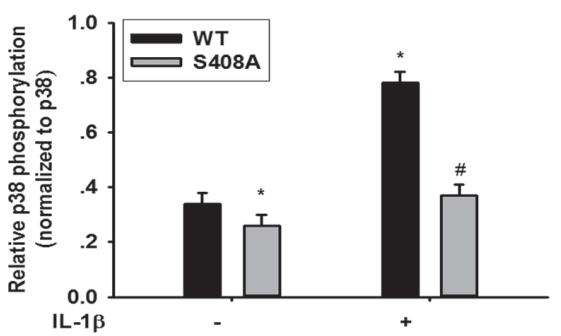

Figure 3: O-GIcNAcylation of TAB3 triggered activation of TAK1 and phosphorylation of its downstream NF- $\mathrm{B}$ and p38 signaling. (A) Stably WT and S408A TAB3 transfected MDA-MB-231 cells were serum starved for $6 \mathrm{~h}$, and then stimulated by IL-1 $\beta$ for $15 \mathrm{~min}$. The TAK1 complexes were pulled down from the cell extracts (1 mg of protein extract) using glutathione-sepharose beads, and TAK1 activity assays were performed as described in the materials and methods section. (B) In parallel to the previous experiments, cell lysates were subjected to SDS-PAGE and immunoblotted with a phospho-specific antibody that recognizes TAK1 phosphorylation (p-TAK1) and with aantibody that recognizes total TAK1. Densitometric analysis was performed for TAK1 phosphorylation and normalized against total TAK1. The data were means \pm SEM. (C) Cell lysate obtained from the previous samples were immunoblotted for phosphorylated

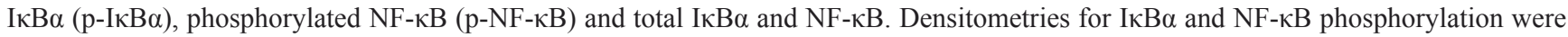
normalized against total $\mathrm{I} \kappa \mathrm{B} \alpha$ and NF- $\kappa \mathrm{B}$ levels. The data were means \pm SEM. (D) Stably transfected cells were further transfected with plasmids encoding the NF- $\kappa$ B luciferase reporter constructs and PRL for $24 \mathrm{~h}$ before stimulated with IL-1 $\beta$. NF- $\kappa \mathrm{B}$ activation were measured and normalized for transfection efficiency using Renilla luciferase as the internal control. The data were means \pm SEM. (E) Cell lysates were immunoblotted for phosphorylated p38 (p-p38) and total p38. Densitometries for p38 phosphorylation were normalized against total $\mathrm{p} 38$ level. The data were means \pm SEM. $* P<0.05$, statistically significant compared with the IL-1 $\beta$ untreated WT TAB3 transfected group; ${ }^{\sharp} P<0.05$, statistically significant compared with IL- $1 \beta$ treated WT TAB3 transfected group. 
cells into the fat pads of mice significantly increased lung and liver metastasis compared with the injection of MDAMB-231 cells. It was noted that the lung metastasis of the S408A mutant TAB3 over-expressing group was less compared with the WT TAB3 over-expressing, although it was still increased compared with the injection of MDAMB-231 cells (Figure 5C-5D).

\section{TAB3 O-GIcNAcylation was correlated with cancer metastasis and poor prognosis in TNBC patients}

To determine whether our findings are clinically relevant, the expression of TAB3 and its O-GlcNAc modification in 280 breast cancer cases were analyzed by immunohistochemistry.
We found that TAB3 and TAB3 O-GlcNAcylation immunoreactivity were identified only in the ER-PRHER2- TNBC cases of breast cancer, but rarely in $\mathrm{ER}+\mathrm{PR}+$ lumina subtype or HER2+ patients, which was similar to the finding in cells (Figure 6A, Table 1). In the $120 \mathrm{TNBC}$ samples, the expression of TAB3 and its O-GlcNAc modification were associated with the tumor grade and the lymph nodes metastases, but was no associated with patients' age and tumor size (Table 2). Univariate analysis was performed to determine whether TAB3 O-GlcNAcylation was a prognostic factor of TNBC. The results show that patients with high TAB3 expression and its O-GlcNAcylation had poorer prognosis than those with low TAB3 expression (Figure 6B). In further, the high O-GlcNAcylation indicated the poorer prognosis in TNBC patients (Figure S6).

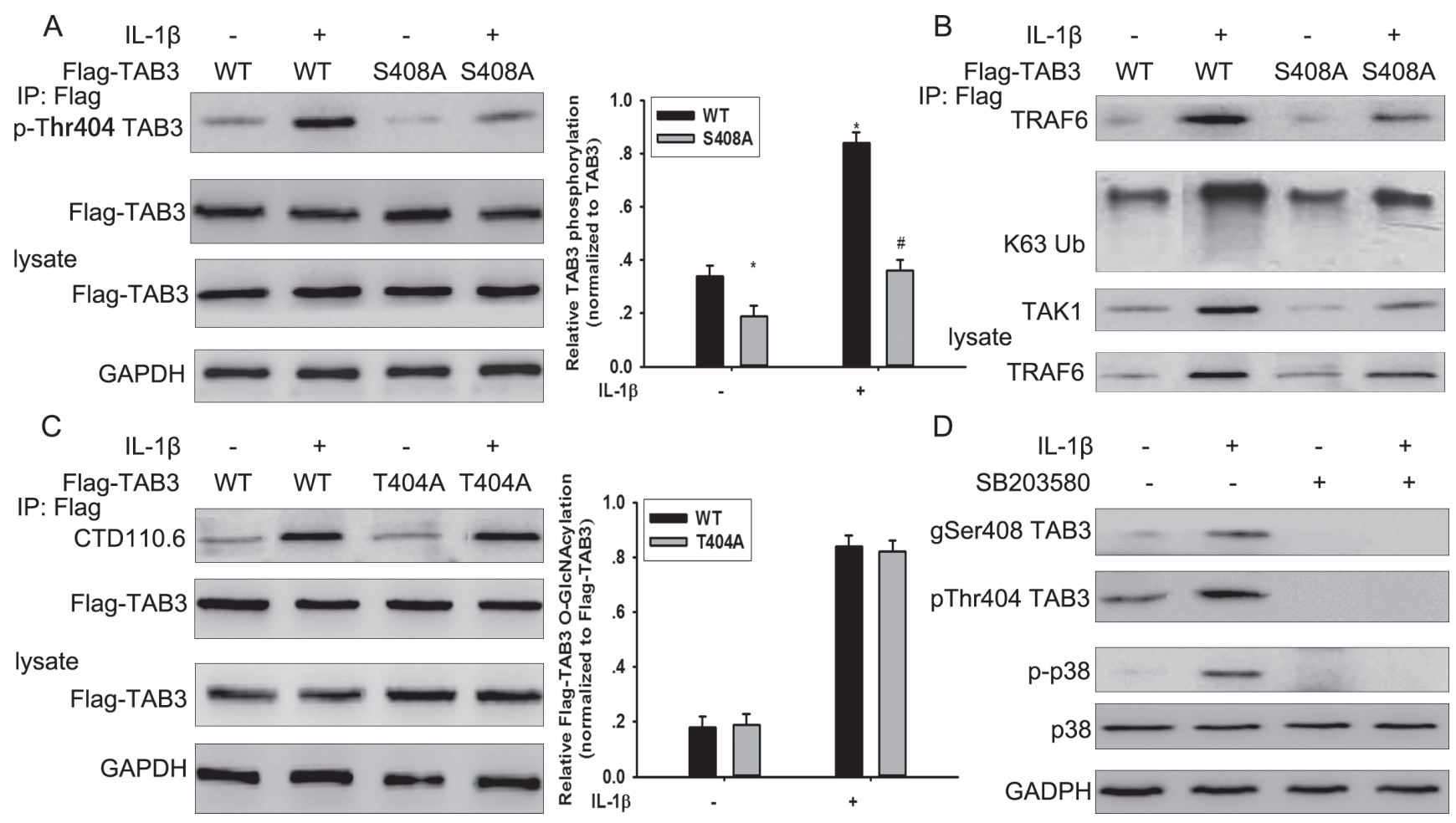

Figure 4: The interplay between TAB3 O-GlcNAcylation and phosphorylation. (A) Stably WT and S408A TAB3 transfected MDA-MB-231 cells were serum starved for $6 \mathrm{~h}$, and then stimulated by IL-1 $\beta$ for $15 \mathrm{~min}$. Aliquots of cell extract were immunoprecipitated with Flag antibody and then subjected to SDS-PAGE and immunoblotted with antibodies that recognize TAB3 phosphorylated at Thr 404. The protein was detected with the Flag antibody as a loading control. Densitometry for Flag-TAB3 Thr 404 phosphorylation was normalized against total Flag-TAB3. The data were means \pm SEM. $* P<0.05$, statistically significant compared with the IL- $1 \beta$ untreated WT TAB3 transfected group; ${ }^{\#} P<0.05$, statistically significant compared with IL-1 $\beta$ treated WT TAB3 transfected group. (B) Immunoprecipitation obtained from the samples previously subjected to SDS-PAGE and immunoblotted with antibodies that recognized TRAF6, TAK1 and K63 linked ubiquitin (K63Ub). (C) Stably WT and T404A TAB3 transfected MDA-MB-231 cells were serum starved for 6 h, and then stimulated by IL-1 $\beta$ for 15 min. Aliquots of cell extract were immunoprecipitated with Flag antibody and then subjected to SDS-PAGE and immunoblotted with CTD110.6 antibody. The protein was detected with the Flag antibody as a loading control. Densitometry for Flag-TAB3 O-GlcNAcylation was normalized against total Flag-TAB3. The data were means \pm SEM. $* P<0.05$, statistically significant compared with the IL-1 $\beta$ untreated WT TAB3 transfected group; ${ }^{\#} P<0.05$, statistically significant compared with IL-1 $\beta$ treated WT TAB3 transfected group. (D) Serum starved MDA-MB-231 cells were pretreated with SB203580 for 5 min, and then stimulated by IL-1 $\beta$ for 15 min. Cell lysate were subjected to SDS-PAGE and immunoblotted with a O-GlcNAc and phospho-specific antibodies that recognizes TAB3 (gSer408 TAB3 antibody and pThr404 TAB3 antibody), phospho-specific antibody that recognizes p38 (p-p38). The detection with total p38 and GAPDH antibodies were used as the loading controls. 
A

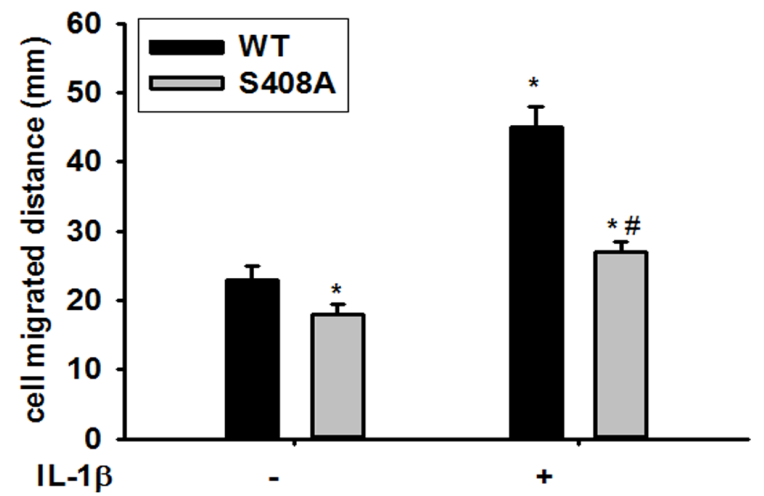

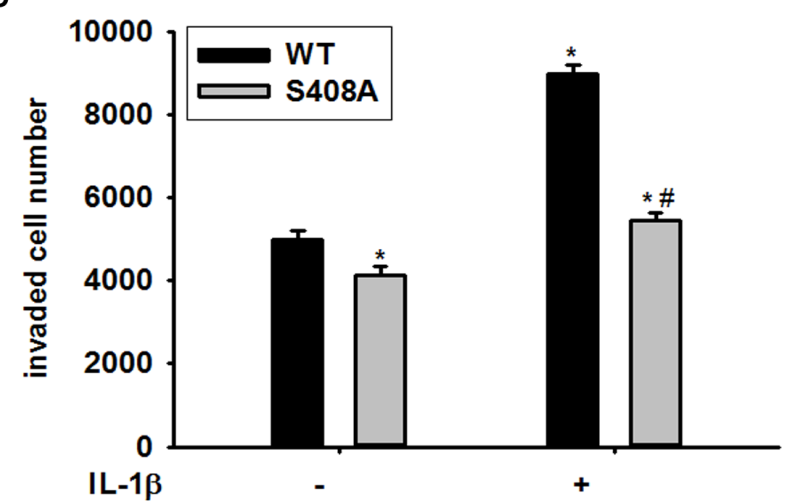

C control WT TAB3 S408A TAB3
B
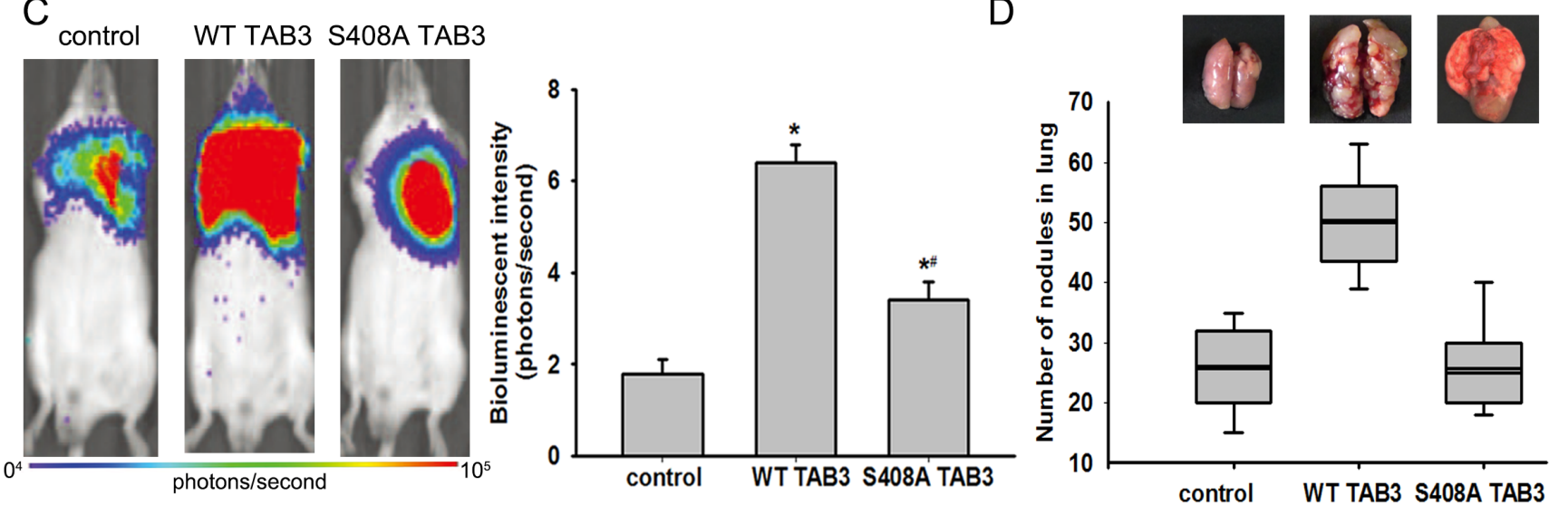

Figure 5: TAB3 O-GlcNAcylation promoted TNBC cell migration and invasion in vitro and in vivo. (A) Equal numbers of WT and S408A TAB3 stably transfected MDA-MB-231 cells seeded into six-well culture plates in the presence or absence of IL-1 $\beta$. Wound healing assay was performed and analyzed. The data were means \pm SEM. $* P<0.05$, statistically significant compared with the IL-1 $\beta$ untreated WT TAB3 transfected group; ${ }^{~} P<0.05$, statistically significant compared with IL-1 $\beta$ treated WT TAB3 transfected group. (B) 106 stably transfected and MDA-MB-231 cells cultured in matrigel chambers with or without IL-1 $\beta$. Transwell assay was performed and analyzed. The data were means \pm SEM. $* P<0.05$, statistically significant compared with the IL-1 $\beta$ untreated WT TAB3 transfected group; ${ }^{*} P<0.05$, statistically significant compared with IL-1 $\beta$ treated WT TAB3 transfected group. (C) WT and S408A TAB3 stably transfected MDA-MB-231 cells were injected into the mammary fat pads of nude mice. The bioluminescent change emitted from the whole bodies of the mice ( 9 mice per group) after repeated intraperitoneal injections of CDDP. The data were means $\pm \mathrm{SEM}$. ${ }^{*} P<0.05$, statistically significant compared with the untransfected group. (D) Representative pictures of murine whole lung The number of visible surface metastatic lesions in mice (9 mice per group) was significantly increased in the WT TAB3 group compared to that in S408A TAB3 group.

A

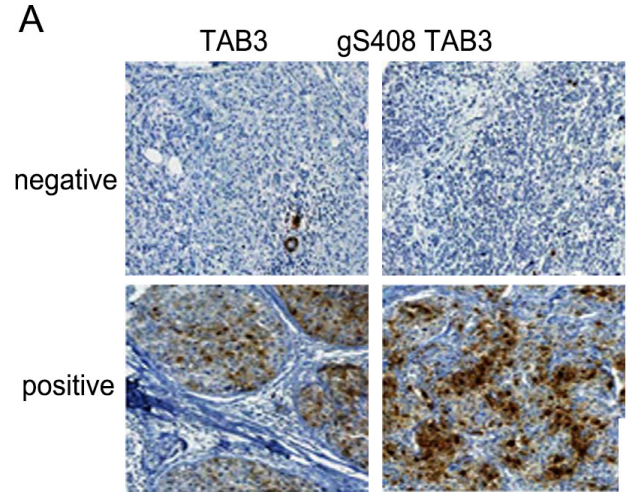

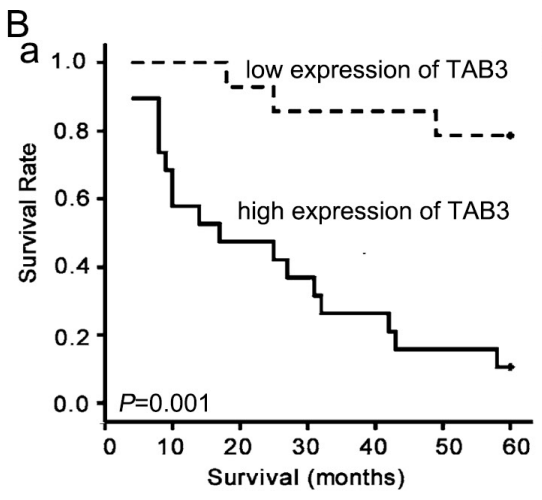

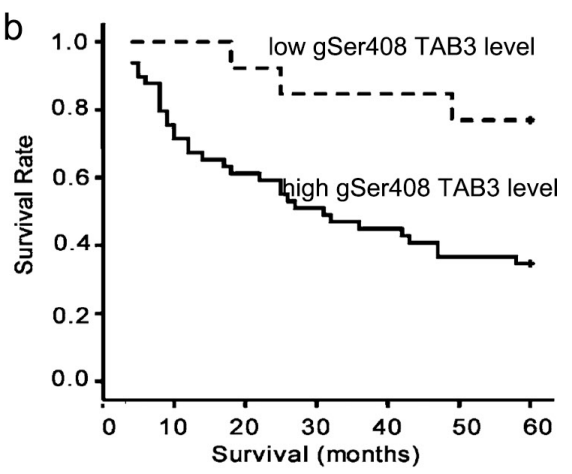

Figure 6: The TAB3 O-GlcNAcylation is correlated with the poorer prognosis in TNBC patients. (A) Representative negative and positive IHC staining for TAB3 expression and O-GlcNAcylated TAB3 in the sections from human breast cancer samples. Scale bars represent $50 \mathrm{~mm}$. (B) Kaplan-Meier survival curve of breast cancer patients with lowand high TAB3 expression (a) and its O-GlcNAcylation (b) $(p<0.001$ by log-rank test). Median follow-up period of 60 months. The number of surviving patients stratified to the follow-up periods is indicated below the graph. 
Table 1: The expression of TAB3 and its O-GIcNAcylation in breast cancer

\begin{tabular}{|c|c|c|c|c|c|c|c|}
\hline \multirow{2}{*}{ Subtype } & \multirow{2}{*}{ Case No. } & \multicolumn{2}{|c|}{ TAB3 } & \multirow{2}{*}{$\boldsymbol{P}$} & \multicolumn{2}{|c|}{ TAB3 O-GIcNAcylation } & \multirow{2}{*}{$\boldsymbol{P}$} \\
\hline & & Positive & Negative & & Positive & Negative & \\
\hline Lumina A & 60 & 0 & 60 & \multirow{4}{*}{$<0.001$} & 0 & 60 & \multirow{4}{*}{$<0.001$} \\
\hline Lumina B & 60 & 0 & 60 & & 0 & 60 & \\
\hline HER-2 & 50 & 0 & 40 & & 0 & 40 & \\
\hline Basal-like (TNBC) & 110 & 118 & 2 & & 78 & 42 & \\
\hline
\end{tabular}

Table 2: Correlation between TAB3 expression and its O-GIcNAcylation and the clinicopathologic features of TNBC

\begin{tabular}{|c|c|c|c|c|c|c|c|}
\hline \multirow{2}{*}{ Criteria } & \multirow{2}{*}{ Case No. } & \multicolumn{2}{|c|}{ TAB3 } & \multirow{2}{*}{$\boldsymbol{P}$} & \multicolumn{2}{|c|}{ TAB3 O-GIcNAcylation } & \multirow{2}{*}{$P$} \\
\hline & & High & Low & & High & Low & \\
\hline \multicolumn{8}{|l|}{ Age } \\
\hline$<50$ & 44 & 20 & 24 & \multirow{2}{*}{0.809} & 18 & 26 & \multirow{2}{*}{0.782} \\
\hline$\geq 50$ & 66 & 24 & 42 & & 22 & 44 & \\
\hline \multicolumn{8}{|l|}{ Size } \\
\hline$\leq 2$ & 72 & 22 & 50 & \multirow{2}{*}{$0.024 *$} & 20 & 52 & \multirow{2}{*}{$0.043 *$} \\
\hline$>2$ & 38 & 22 & 16 & & 21 & 17 & \\
\hline \multicolumn{8}{|l|}{ Grade } \\
\hline I & 50 & 7 & 33 & \multirow{3}{*}{0.145} & 5 & 35 & \multirow{3}{*}{0.097} \\
\hline II & 45 & 24 & 21 & & 21 & 24 & \\
\hline III & 15 & 13 & 2 & & 10 & 5 & \\
\hline \multicolumn{8}{|c|}{ Axillary lymph node status } \\
\hline N0 & 30 & 7 & 23 & \multirow{3}{*}{$0.001 *$} & 5 & 25 & \multirow{3}{*}{$0.013 *$} \\
\hline N1 & 48 & 18 & 30 & & 16 & 32 & \\
\hline N2 & 32 & 18 & 14 & & 17 & 15 & \\
\hline
\end{tabular}

\section{DISCUSSION}

Tumor metastasis is the major cause of death in TNBC [20]. Increasing evidence have suggested that activation of $\mathrm{NF}-\kappa \mathrm{B}$ by the inflammatory microenvironments promote the TNBC metastasis [21]. The present study found that a novel NF- $\kappa$ B regulator TBA3 was modified by O-GlcNAcat Ser408, and its O-GlcNAcylation is correlated with TNBC metastasis regulated by the NF- $\kappa \mathrm{B}$ signaling pathway.

Previous studies have shown that increased levels of global O-GlcNAcylation and O-GlcNActransferase are linked to the incidence of metastasis in breast cancer patients [22]. However, whether there was a specific substrate of OGT in the TNBC was unknown. The TABs family consists of three members, including TAB1, TAB2 and TAB3. TAB1 was reported to have O-GlcNAcylation and involved in NF- $\kappa \mathrm{B}$ activation in macrophages. It was not expressed in breast cancer [7]. TAB2 and TAB3were reported to be able to form complex with TAK1, which induced breast cancer metastasis [18]. However, we found that TAB2 could not be O-GlcNAcylatedby OGT (Data not shown). Therefore, we focused on the role of TAB3 $\mathrm{O}-$ GlcNAcylation in breast cancer.

As the key modulator of NF- $\kappa \mathrm{B}$, TAB3 played an essential role in immunity, such as anti-virus infection, B cell activation and endotoxin shock [23, 24]. Recently, it 
was reported that miR-195 inhibited cell survival, migration and invasion by targeting TAB 3 expression in hepatocellular carcinoma [25]. We have found TAB3 specifically expressed in TNBC, and it suppressed cell migration and invasion. TAB3 could bind to the TRAFs protein through their C-terminal nuclear zinc finger (NZF) motif, which leads to the autophosporylation and activation of TAK1 and its downstream NF- $\mathrm{BB}$ signal molecule [26]. TAB3 was phosphorylated by p38 MAPK at Ser60, Thr404 and Ser506, and also involved in TAK1 activation. In identifying the phosphorylation sites of TAB3, the molecular mass of the peptides NQHSLYTATTPPSSSPSR was 203 Da greater than the theoretical value, which equates to the mass predicted for a residue attached covalently to a serine or threonine residue in the peptide [8]. We found that Ser408 is the only O-GlcNAcylated site in TAB3. In further, TAB3 O-GlcNAcylation was essential in cancer metastasis both in vivo and in vitro. These data suggested thatTAB3 was a specific substrate of OGT, and played an essential role in OGT mediated cancer metastasis in the TNBC.

Given that O-GlcNAcylation and phosphorylation both occur at serine and threonine residues, the reciprocal relationship between O-GlcNAcylation and O-phosphorylation gives rise to the most acceptable "yin-yang hypothesis" for understanding the function of O-GlcNAcylation [27]. We have found that the O-GlcNAcylation site Ser 408 is close to the phosphorylation site Thr404 of TAB3. The O-GlcNAcylation and phosphorylation at proximal residues of the same protein affect each other via spatial interactions. Abolish of O-GlcNAcylation at Ser 408 reduced Thr404 phosphorylation in TAB3. However, Ala mutation of Thr404, which disturbed the phosphorylation, has no effect on the O-GlcNAcylation. This result suggested that the O-GlcNAcylation promote phosphorylation in TAB3. Previous study has demonstrated that the phosphorylation of TAB3 is mediated by $\mathrm{p} 38$ MAPK during IL-1 $\beta$ activation [18]. And p38 MAPK activate neuronal proteins O-GlcNAcylation in glucose deprivation [28]. Our finding shown the TAB3 O-GlcNAcylation was suppressed when cells were pretreated with the p38MAPK inhibitor SB203580. Furthermore, TAB3O-GlcNAcylationalso promoted TAK1 downstream p38 MAPK signal activation [6, 29]. It indicated that the $\mathrm{p} 38 \mathrm{MAPK}$ activation promoted the TAB3 O-GlcNAcylation and it in turn mediated TAB3 Thr404 phosphorylation in TNBC, The O-GlcNAcylation of TAB3 enhanced the TAK1 mediated p38MAPK activation, which formed the positive feedback loop and promoted the NF- $\kappa \mathrm{B}$ activation and TNBC metastasis.

In conclusion, the present study revealed the biological significance of TAB3 O-GlcNAcylation in TNBC and illustrated that TAB3 O-GlcNAcylation promoted its metastasis by triggering TAK1 mediated NF- $\mathrm{B}$ activation. These findings represented a possible mechanism in inflammatory induced cancer cell metastasis and provided a strategy for the development of novel therapies to prevent tumor metastasis in TNBC.

\section{MATERIALS AND METHODS}

\section{Plasmids construction}

The full length human TAB3 (GenBank No. NM_152787.4) and OGT (GenBank No. NM_181672.2) cDNA were obtained from the fetal liver cDNA library. The GST-TAB3, Flag-TAB3, HA-OGT and Flag-OGT were generated by standard procedures as previously described [30]. The sequences of RNAi, shRNA targeting the TAB3 (TCCACAGCATCAAGTGCAACC) and OGT (CAAGTGTACTGCAGCAGCAGG) were cloned into the pCDNA6.2-GW/EGFP-miR vector per the manufacturers' instruction (Invitrogen, Carlsbad, USA). The TAB3 O-GlcNAcylation site mutant (S408A) and TAB3 phosphorylation site mutant (T404A) vectors were constructed using the sequence overlap extension PCR. Primers used in this study were shown in Supplementary Information Table S1.

\section{Cell culture, transfection and stimulation}

Human breast cancer cell lines $\mathrm{MCF}-7(\mathrm{ER}+/ \mathrm{PR}+/$ HER2-, Luminal A subtype), T47D (ER+/PR+/HER2+, Luminal B subtype), SK-BR-3 (ER-/PR-/HER2+, HER2+ subtype), MDA-MB-231 (ER-/PR-/HER2-, Basel-like subtype), and MDA-MB-468 (ER-/PR-/HER2-, Basellike subtype) were obtained from the Cell Resource Center, Shanghai Institute for Biology Science, Chinese Academy of Sciences (Shanghai, China). These cells were cultured as previously described [31]. Transient transfections were performed using lipofectamine 2000 following manufacturer's recommendation. For the creation of stable cells, transfected cells were exposed to $0.5 \mathrm{mg} / \mathrm{ml}$ neomycin (for over-expression vector) or $0.01 \mathrm{mg} / \mathrm{ml}$ blasticidin (for RNAi vector) for 2 weeks. Prior to stimulation with human IL- $\beta$, the medium was removed and replaced with fresh medium from which FCS had been omitted for $6 \mathrm{~h}$. GlcNAcstatin $(1 \mathrm{mM})$ was added to cells during serum starvation, if required.

\section{Cell migration and invasion assay}

Wound healing assay was used to detect cell migration ability. Briefly, equal numbers of stably transfected cells were seeded into six-well culture plates. When the cells reached $90 \%$ confluence, a scratch wound was created. The migrated distances of the cells into the wounded area was calculated by subtracting the distance from the initial distance 24 hours after wound healing. To detect the cell invasion ability, $10^{6}$ stable transfected cells were 
sub-cultured on the transwell filter coated with Matrigel. After incubation for 24 hours, cells on the upper chamber were stained and counted under an inverted microscope.

\section{In vitro $\mathrm{O}-\mathrm{GlcNAc}$ assay, enzymatic labeling of O-GIcNAc sites, and $\beta$-Elimination}

In vitro O-GlcNAcylation of TAB3 was detected as previously described [8]. The TAB3 $(1 \mathrm{mM})$ was added into $20 \mu \mathrm{l}$ reaction buffer $(50 \mathrm{mMTris}-\mathrm{HCl}[\mathrm{pH}=7.5], 1 \mathrm{mM}$ DTT, $12.5 \mathrm{mM} \mathrm{MgCl}_{2}$ ) which contain $50 \mathrm{mM}$ OGT and $1 \mathrm{mM}$ UDP-GlcNAc. The reaction mixtures were incubated for $90 \mathrm{~min}$ at $37^{\circ} \mathrm{C}$, stopped by adding loading buffer, resolved on Western blot with appropriate antibodies. To enzymatic label TAB3 at O-GlcNAc site, O-GlcNAcylated GST-TAB3 bound beads and was labeled using Click$\mathrm{iT}^{\mathrm{TM}}$ O-GlcNAc enzymatic labeling system (Invitrogen, Carlsbad, USA) and detected by Click-i $\mathrm{T}^{\mathrm{TM}}$ biotin protein analysis detection kit (Invitrogen, Carlsbad, USA) following manufacturer's recommendation. $\beta$-elimination was performed overnight at $4^{\circ} \mathrm{C}$ using the GlycoProfile $\beta$-elimination kit (Sigma, St. Louis, USA) according to the manufacturer's instructions.

\section{O-GlcNAc site mapping of TAB3}

For site mapping analysis of digested TAB3 protein, purified FLAG-TAB3 was reduced, alkylated, and enzymatically digested with trypsin. An aliquot of the digested sample was loaded onto a capillary precolumn (360 $\mu \mathrm{m}$ outer diameter $* 75 \mu \mathrm{m}$ inner diameter, Polymicro Technologies) packed with $\mathrm{C} 18$ reverse-phase resin (20 $\mu \mathrm{m}$ diameter, $120 \AA$ pore size, YMC Inc.). The precolumn was rinsed with $0.1 \%$ acetic acid to remove salts and connected to a capillary analytical column $(360 \mu \mathrm{m}$ outer diameter * $50 \mu \mathrm{m}$ inner diameter, Polymicro Technologies) packed with $\mathrm{C} 18$ resin $(5-20 \mu \mathrm{m}$ diameter, $120 \AA$ A pore size, YMC Inc.). The analytical column was equipped with an integrated electrospray emitter. Proteolytic peptides were gradient eluted into the mass spectrometer at $60 \mathrm{nl} / \mathrm{min}$ flow rate using the high pressure liquid chromatography. Samples were analyzed on a LTQ-Orbitrap mass spectrometer (Thermo Fisher Scientific, USA) where the Orbitrap analyzer was operated at a resolving power of 30,000 (at $\mathrm{m} / \mathrm{z} 400$ ) to acquire high resolution MS1 spectra. Collision-activated dissociation (CAD) mass spectra were acquired data-dependently using the quadrupole linear ion trap analyzer. An LTQ XL mass spectrometer (Thermo Fisher Scientific, USA) was used to acquire electron transfer dissociation (ETD) mass spectra. For ETD analyses (reagent AGC target $=3 * 10^{5}$ ion counts, ETD reaction time $=100 \mathrm{~ms}$, precursor isolation window $=4 \mathrm{~m} / \mathrm{z}$ ), the LTQ XL was operated to continuously cycle through a MS1 scan followed by an ETD scan recorded on m/z 1318.8 followed by four data-dependent ETD scans. All data were interpreted manually.

\section{Immunoprecipitation and western blot}

For immunoprecipitation assays, cells were collected in PBS and lysed by RAPI buffer. After preclearing with protein $\mathrm{G}$ sepharose beads, cell lysates were incubated with specific antibody bound to either protein $\mathrm{A} / \mathrm{G}$ sepharose beads for 12 hours at $4^{\circ} \mathrm{C}$. Precipitated immune-complexes were washed with RIPA buffer four times, eluted by boiling in $2 \times$ SDS sample buffer, resolved by SDS-PAGE gel, and analyzed by immunoblotting as previously reported [32].

\section{TAK1 activity assays}

The TAK1 activities were detected as previously reported [33]. Briefly, the TAK1 complexes were pulled down using the glutathione-sepharose beads from cell lysate at $4^{\circ} \mathrm{C}$ for $2 \mathrm{~h}$. The beads were washed twice with $1 \mathrm{ml}$ of lysis buffer containing $0.25 \mathrm{MNaCl}$, followed by two washes with high salt wash buffer ( $1 \mathrm{ml}$ of $50 \mathrm{mM}$ Tris- $\mathrm{HCl} \mathrm{pH} 7.5,0.5 \mathrm{M} \mathrm{NaCl}$ and $0.1 \%$ 2-mercaptoethanol). TAK1 activity was assayed by its ability to activate MKK6, as judged by the activation of SAPK2a/p38a. The active SAPK2a/p38a generated in this first stage of assay was then quantitated in a second assay by measuring phosphorylation of myelin basic protein.

\section{NF-кB luciferase reporter assay}

To assess transcriptional activity of NF- $\kappa \mathrm{B}$, cells were transiently transfected with $0.5 \mu \mathrm{g}$ of PRL-SV40 vector encoding Renilla luciferase and $0.5 \mu \mathrm{g}$ of encoding the $3 * \kappa B$ luciferase reporter PGL-3 vector construct. After $24 \mathrm{~h}$, the cells were stimulated with IL-1 $\beta$ for $24 \mathrm{~h}$ and then the cells were lysed. The luciferase activity was then measured using a Dual-Luciferase Reporter Assay System (Promega, Madison, USA) as per the manufacturer's instructions. Firefly luciferase activity was normalized by Renilla luciferase activity for each transfection.

\section{Pathological samples and immunohistochemistry assay}

All investigations described in this study were performed after informed consent was obtained and in accordance with an institutional review board protocol approved by the Partners Human Research Committee at Fudan University Shanghai Cancer Center. Breast cancer tissues were obtained from the Department of Pathology at Fudan University Shanghai Cancer Center from 2005 to 2009 under the auspices of an institutional review board approved human subjects study protocol. Formalin-fixed, paraffin embedded sections were stained by immunochemistry with standard procedures as previously reported [34]. The intensity of immunostaining in each tumor section was assessed as strong (3), moderate (2), 
weak (1), or negative (0); semiquantitatively using the following scale: $<5 \%$ of cells (0), 5-25\% (1), 26-50\% (2), $50-75 \%$ (3), and $>75 \%$ (4) of cells, and then combined these values. This resulted in an overall TAB3 expression and O-GlcNAcylation immunohistochemical score ranging from 0 to 12 . The value was considered high when scores were $>3$, and low when scores were $<$ 3. Immunohistochemical evaluation for estrogen receptor (ER), progesterone receptor (PR), and HER-2 was performed by three pathologists in the Department of Pathology, Fudan University Shanghai Cancer Center.

\section{Mouse model for breast cancer metastasis}

All animal works were conducted in accordance with a protocol approved by the Institutional Animal Care and Use Committee at the Shanghai medical college of Fudan University. Female nude mice (3 to 4 weeks old) were injected with stably transfected $10^{7}$ MDA-MB-231 cells into the mammary fat pads of the mice. Tumor growth was evaluated by monitoring tumor volume every 3 days. The whole-body metastasis of animals bearing xenografts that stably express luciferase was monitored using the IVIS Lumina Imaging System (Xenogen, USA) every 2 days. The animals were sacrificed when the xenografts reached $1.5 \mathrm{~cm}$ in diameter. The tumor xenografts and lungs of the sacrificed mice were harvested for further investigation.

\section{Statistical analysis}

All experiments were repeated at least three times. All numerical data were described as mean \pm SEM. Data was analyzed using the two-tailed $t$-test. A probability value of 0.05 or less was considered significant. Tests for association between TAB3 expressions or its O-GlcNAcylation and clinicopathological variables were computed using the Mantel-Haenzel $\chi^{2}$ test or Fisher exact test. Survival estimates were computed using the Kaplan-Meier method, and comparisons between groups were analyzed using the log-rank test. The variables achieving significance at $\alpha=0.05$ level was included.

\section{GRANT SUPPORT}

The work was supported by the NST (Grants 2012CB910602 and 2012AA020203), NSF (Grants 21335002), the Ph.D. Programs Foundation of Ministry of Education of China (20130071110034) and Shanghai Projects (Eastern Scholar, 15JC1400700 and B109).

\section{CONFLICTS OF INTEREST}

The authors declare no conflicts of interest.

\section{REFERENCES}

1. Fan L, Strasser-Weippl K, Li JJ, St Louis J, Finkelstein DM, Yu KD, Chen WQ, Shao ZM, Goss PE. Breast cancer in China. Lancet Oncol. 2014; 15:e279-289.

2. Jiang X, Shapiro DJ. The immune system and inflammation in breast cancer. Mol Cell Endocrinol. 2014; 382:673-682.

3. Perrier S, Caldefie-Chezet F, Vasson MP. IL-1 family in breast cancer: potential interplay with leptin and other adipocytokines. FEBS Lett. 2009; 583:259-265.

4. Li Q, Verma IM. NF-kappaB regulation in the immune system. Nat Rev. 2002; 2:725-734.

5. Prabhu L, Mundade R, Korc M, Loehrer PJ, Lu T. Critical role of NF-kappaB in pancreatic cancer. Oncotarget. 2014; 5:10969-10975. doi: 10.18632/oncotarget.2624.

6. Bradley JR, Pober JS. Tumor necrosis factor receptorassociated factors (TRAFs). Oncogene. 2001; 20:6482-6491.

7. Safina A, Sotomayor P, Limoge M, Morrison C, Bakin AV. TAK1-TAB2 signaling contributes to bone destruction by breast carcinoma cells. Mol Cancer Res. 2011; 9:1042-1053.

8. Ishitani T, Takaesu G, Ninomiya-Tsuji J, Shibuya H, Gaynor RB, Matsumoto K. Role of the TAB2-related protein TAB3 in IL-1 and TNF signaling. EMBO J. 2003; 22:6277-6288.

9. Hart GW, Slawson C, Ramirez-Correa G, Lagerlof O. Cross talk between O-GlcNAcylation and phosphorylation: roles in signaling, transcription, and chronic disease. Annu Rev Biochem. 2011; 80:825-858.

10. Hart GW, Kreppel LK, Comer FI, Arnold CS, Snow DM, Ye Z, Cheng X, DellaManna D, Caine DS, Earles BJ, Akimoto Y, Cole RN, Hayes BK. O-GlcNAcylation of key nuclear and cytoskeletal proteins: reciprocity with O-phosphorylation and putative roles in protein multimerization. Glycobiology. 1996; 6:711-716.

11. Liu Y, Liu F, Grundke-Iqbal I, Iqbal K, Gong CX. Brain glucose transporters, O-GlcNAcylation and phosphorylation of tau in diabetes and Alzheimer's disease. J Neurochem. 2009; 111:242-249.

12. Gu Y, Mi W, Ge Y, Liu H, Fan Q, Han C, Yang J, Han F, $\mathrm{Lu} \mathrm{X}, \mathrm{Yu}$ W. GlcNAcylation plays an essential role in breast cancer metastasis. Cancer Res. 2010; 70:6344-6351.

13. Hanover JA, Krause MW, Love DC. Bittersweet memories: linking metabolism to epigenetics through O-GlcNAcylation. Nat Rev Mol Cell Bio. 2012; 13:312-321.

14. Ferrer CM, Lynch TP, Sodi VL, Falcone JN, Schwab LP, Peacock DL, Vocadlo DJ, Seagroves TN, Reginato MJ. O-GlcNAcylation regulates cancer metabolism and survival stress signaling via regulation of the HIF-1 pathway. Mol Cell. 2014; 54:820-831.

15. Khidekel N, Arndt S, Lamarre-Vincent N, Lippert A, PoulinKerstien KG, Ramakrishnan B, Qasba PK, Hsieh-Wilson LC. 
A chemoenzymatic approach toward the rapid and sensitive detection of O-GlcNAc posttranslational modifications. J Am Chem Soc. 2003; 125:16162-16163.

16. Dorfmueller HC, Borodkin VS, Schimpl M, Shepherd SM, Shpiro NA, van Aalten DM. GlcNAcstatin: a picomolar, selective O-GlcNAcase inhibitor that modulates intracellular O-glcNAcylation levels. J Am Chem Soc. 2006; 128: 16484-16485.

17. Jin G, Klika A, Callahan M, Faga B, Danzig J, Jiang Z, Li X, Stark GR, Harrington J, Sherf B. Identification of a human NF-kappaB-activating protein, TAB3. Proc Natl Acad Sci U S A. 2004; 101:2028-2033.

18. Mendoza H, Campbell DG, Burness K, Hastie J, Ronkina N, Shim JH, Arthur JS, Davis RJ, Gaestel M, Johnson GL, Ghosh S, Cohen P. Roles for TAB1 in regulating the IL-1dependent phosphorylation of the TAB3 regulatory subunit and activity of the TAK1 complex. Biochem J. 2008; 409:711-722.

19. Besse A, Lamothe B, Campos AD, Webster WK, Maddineni U, Lin SC, Wu H, Darnay BG. TAK1-dependent signaling requires functional interaction with $\mathrm{TAB} 2 / \mathrm{TAB} 3$. J Biol Chem. 2007; 282:3918-3928.

20. Sarvaiya PJ, Guo D, Ulasov I, Gabikian P, Lesniak MS. Chemokines in tumor progression and metastasis. Oncotarget. 2013; 4:2171-2185. doi: 10.18632/oncotarget.1426.

21. Mitre-Aguilar IB, Cabrera-Quintero AJ, Zentella-Dehesa A. Genomic and non-genomic effects of glucocorticoids: implications for breast cancer. Int J Clin Exp Pathol. 2015; 8:1-10.

22. Netsirisawan P, Chokchaichamnankit D, Srisomsap C, Svasti J, Champattanachai V. Proteomic Analysis Reveals Aberrant O-GlcNAcylation of Extracellular Proteins from Breast Cancer Cell Secretion. Cancer Genom Proteom. 2015; 12:201-209.

23. Lei X, Han N, Xiao X, Jin Q, He B, Wang J. Enterovirus $713 \mathrm{C}$ inhibits cytokine expression through cleavage of the TAK1/TAB1/TAB2/TAB3 complex. J Viol. 2014; 88: 9830-9841.

24. Hong S, Lim S, Li AG, Lee C, Lee YS, Lee EK, Park SH, Wang XJ, Kim SJ. Smad7 binds to the adaptors TAB2 and TAB3 to block recruitment of the kinase TAK1 to the adaptor TRAF2. Nat Immunol. 2007; 8:504-513.
25. Ding J, Huang S, Wang Y, Tian Q, Zha R, Shi H, Wang Q, Ge C, Chen T, Zhao Y, Liang L, Li J, He X. Genome-wide screening reveals that miR-195 targets the TNF-alpha/NFkappaB pathway by down-regulating IkappaB kinase alpha and TAB3 in hepatocellular carcinoma. Hepatology. 2013; 58:654-666.

26. Kanayama A, Seth RB, Sun L, Ea CK, Hong M, Shaito A, Chiu YH, Deng L, Chen ZJ. TAB2 and TAB3 activate the NF-kappaB pathway through binding to polyubiquitin chains. Mol Cell. 2004; 15:535-548.

27. Zeidan Q, Hart GW. The intersections between O-GlcNAcylation and phosphorylation: implications for multiple signaling pathways. J Cell Sci. 2010; 123:13-22.

28. Cheung WD, Hart GW. AMP-activated protein kinase and p38 MAPK activate O-GlcNAcylation of neuronal proteins during glucose deprivation. J Biol Chem. 2008; 283:13009-13020.

29. Yamashita M, Fatyol K, Jin C, Wang X, Liu Z, Zhang YE. TRAF6 mediates Smad-independent activation of JNK and p38 by TGF-beta. Mol Cell. 2008; 31:918-924.

30. Tao T, Cheng C, Ji Y, Xu G, Zhang J, Zhang L, Shen A. Numbl inhibits glioma cell migration and invasion by suppressing TRAF5-mediated NF-kappaB activation. Mol Biol Cell. 2012; 23:2635-2644.

31. Hong Q, Yu S, Yang Y, Liu G, Shao Z. A polymorphism in JMJD2C alters the cleavage by caspase-3 and the prognosis of human breast cancer. Oncotarget. 2014; 5:4779-4787. doi: 10.18632/oncotarget.2029.

32. Tao T, Ji Y, Cheng C, Yang H, Liu H, Sun L, Qin Y, Yang J, Wang H, Shen A. Tumor necrosis factor-alpha inhibits Schwann cell proliferation by up-regulating Src-suppressed protein kinase C substrate expression. J Neurochem. 2009; 111:647-655.

33. Pathak S, Borodkin VS, Albarbarawi O, Campbell DG, Ibrahim A, van Aalten DM. O-GlcNAcylation of TAB1 modulates TAK1-mediated cytokine release. EMBO J. 2012; 31:1394-1404.

34. Huang Q, He X, Qiu X, Liu X, Sun G, Guo J, Ding Z, Yang L, Ban N, Tao T, Wang D. Expression of NF45 correlates with malignant grade in gliomas and plays a pivotal role in tumor growth. Tumour Biol. 2014; 35:10149-10157. 